\section{Functional Components of Fish}

\author{
Nasopoulou Constantina* \\ Department of Food Science and Nutrition, University of the Aegean, \\ Greece
}

Functional foods have not yet been defined by European legislation, even though Regulation (EC) No 1924/2006 of the European Union on nutrition and health claims on foods, referring among others to functional foods, came into force in 2006. Generally, functional foods are considered to be foodstuffs that contain biologically active constituents that contribute to human health maintenance and/or reduce susceptibility to pathological conditions leading to diseases. Fish are inherently functional food (natural functional food) due to the fact that they naturally contain a variety of bioactive constituents such as polyunsaturated omega- 3 fatty acids [1,2], anti-thrombotic lipid micro-constituents [3,4], bioactive peptides [5], vitamin $\mathrm{D}_{3}[6,7]$ and minerals $[8,9]$.

Polyunsaturated fatty acids are classed-according to the position of the first double bond-in omega- 3 and omega- 6 fatty acids. Human body lacks the necessary enzymes to convert omega- 6 to omega- 3 fatty acids, therefore the latter must be obtained from the diet. The main omega-3 polyunsaturated fatty acids derived from fish oil are Eicosapentaenoic Acid (EPA) and Docosahexaenoic Acid (DHA). EPA and DHA have been related to several cardioprotective properties such as anti-inflammatory, anti-thrombotic and anti-atherosclerotic activity, along with improving endothelial function and decreasing blood pressure and triacylglycerides concentration, even though the mechanisms of action remain unclear [2]. More specific a suggested mechanism of the anti-inflammatory activity of EPA and DHA could be due to the fact that consumption of omega-3 fatty acids increases eicosapentaenoic acid in the cell membrane, which antagonise the pro-inflammatory activity of the omega- 6 eicosanoids, while a proposed mechanism of action related to the anti-atherosclerotic effect of EPD and DHA may be attributted to inflammation reduction, attenuation of growth factor production, or suppression of smooth muscle cell proliferation [2]. Additionally DHA is the most abundant omega- 3 fatty acid in the mammalian brain membranes and nervous system and its levels in brain membrane lipids and nervous tissue are altered according to the type and content of fatty acids in the

*Corresponding author: Nasopoulou Constantina, Department of Food Science and Nutrition, University of the Aegean, 2 Mitropoliti loakim St, 81400, Myrina, Lemnos, Greece, Tel: +30 2254083124; E-mail: knasopoulou@aegean.gr

Citation: Nasopoulou C (2016) Functional Components of Fish. J Food Sci Nutr 2: 009.

Received: March 15, 2016; Accepted: March 25, 2016; Published: April 11, 2016 diet. Therefore DHA is essential for cognitive development, neurogenesis, neurotransmission and protection against oxidative stress [10], while vitamin D along with DHA or EPA may attenuate the severity of hyperactivity disorder, depression, bipolar disorder and schizophrenia by regulating serotonin synthesis [11].

According to some researchers, it is believed that the cardioprotective properties of fish oil could be attributed not only to the presence of omega-3 polyunsaturated fatty acids but also to the presence of some other micro-constituents $[12,13]$. Fish oil have been found to contain polar lipid constituents with chemical structure similar to phosphatidylcholine derivatives [14] that possess both in vitro anti-thrombotic $[3,4]$ and in vivo anti-atherogenic properties [15]. The anti-thrombotic activity of fish polar lipids is mainly due to the inhibition of Platelet Activating Factor (PAF)-induced platelet activation and aggregation [3,4], while the mechanism of the anti-atherogenic activity of fish polar lipids is mainly attributed both to reduction of the enzymatic activity of PAF-Cholinephosphotransferase (PAF-CPT); a biosynthetic enzyme of PAF and increase of the enzymatic activity of PAF-Acetylhydrolase (PAF-AH); a catabolic enzyme of PAF [16].

Fish bioactive peptides have been found to display several biological properties such as antioxidant, anti-hypertensive, anti-thrombotic, antimicrobial, immunomodulatory, prebiotic, mineral binding and hypocholesterolemic activity. The aforementioned biological properties of fish peptides depend on different factors including their structural properties, amino acid composition and amino acid sequences [17]. More specific the antioxidant activity of fish bioactive peptides is attributed to the presence of hydrophobic amino acids, aromatic amino acids and histidine; the anti-hypertensive activity is suggested to be due to the potent inhibitory activity of fish peptides against angiotensin-I converting enzyme, which plays a crucial role in blood pressure regulation by converting angiotensin-I to angiotensin-II - a potent vasoconstrictor-while the hypocholesterolemic effect of fish protein hydrolysate is exerted by in vivo reducing plasma cholesterol level and inhibiting the activity of acyl-CoA:cholesterol acyltransferase [5]. Aditionally fish proteins are good source of essential amino acids such as lysine, methionine, cysteine, threonine and tryptophan [18]. Lysine has been recognized as an important precursor for the de novo synthesis of glutamate, the most crucial neurotransmitter in the mammalian central nervous system [19]. Furthermore lysine has been found to enhance intestinal calcium absorption and improve the renal conservation of the absorbed calcium [20], while deficiency or low supply of this compound in the diet may lead to mental and physical handicaps such as osteoporosis.

Fish and fish liver oil are regarded to be the most significant dietary saurces of naturally occuring cholecalciferol (vitamin $\mathrm{D}_{3}$ ). Since sun exposure of humans-following the Western lifestyle-is limited, dietary intake of vitamin $\mathrm{D}_{3}$ is of great importance. Some of the functions of vitamin $\mathrm{D}_{3}$ is regulation of calcium and phosphorus metabolism in order to ensure normal mineralization of bones and perticipation in muscle development, since vitamin D receptor is located in muscle tissue [21]. 
Another functional component of fish is Selenium (Se). Se behaves both as an antioxidant and anti-inflammatory agent. The antioxidant capacity of Se is due to the ability to (i) reduce hydrogen peroxide, lipid and phospholipid hydroperoxides, by blocking the propagation of free radicals and reactive oxygen species, and (ii) reduce hydroperoxide intermediates in the cyclooxygenase and lipoxygenase pathways leading to inflammatory prostaglandins and leukotrienes. Any condition associated with increased levels of oxidative stress or inflammation (e.g., atherosclerosis) might be expected to be influenced by Se levels [22]. Moreover Se seems to be of great importance regarding immune system function since immune system cells (e.g., T-cells) have a functional need for Se [22]. At the same time Se reduce the toxicity of a number of metals (e.g., mercury or methyl mercury in marine foods) by forming inert metal selenide complexes [22].

In recent years, utilisation of marine by-products and isolation of bioactive constituents gave rise to increasing interest. For instance antioxidant peptides obtained by enzymatic hydrolysis of fish proteins have been used as functional components and nutraceuticals since they exhibit not only nutritional but also biological properties for use in diet or in therapeutic purposes [23].

The overall conclusion is that fish and fish products contain naturally a wide range of functional components that can be obtained either by diet or by utilisation of fish by-products.

\section{References}

1. Kris-Etherton PM, Harris WS, Appel LJ, American Heart Association Nutrition Committee (2002) Fish consumption, fish oil, omega-3 fatty acids, and cardiovascular disease. Circulation 106: 2747-2757.

2. Din JN, Newby DE, Flapan AD (2004) Omega 3 fatty acids and cardiovascular disease--fishing for a natural treatment. BMJ 328: 30-35.

3. Nasopoulou C, Psani E, Sioriki E, Demopoulos CA, Zabetakis I (2013) Evaluation of Sensory and in vitro Cardio Protective Properties of Sardine (Sardina pilchardus): The Effect of Grilling and Brining. Food Nutr Sci 4: 940-949.

4. Nasopoulou C, Nomikos T, Demopoulos CA, Zabetakis I (2007) Comparison of antiatherogenic properties of lipids obtained from wild and cultured sea bass (Dicentrarchus labrax) and gilthead sea bream (Sparus aurata). Food Chem 100: 560-567.

5. Ngo DH, Vo TS, Ngo DN, Wijesekara I, Kim SK (2012) Biological activities and potential health benefits of bioactive peptides derived from marine organisms. Int J Biol Macromol 51: 378-383.

6. Schmid A, Walther B (2013) Natural vitamin D content in animal products. Adv Nutr 4: 453-462.

7. Lu Z, Chen TC, Zhang A, Persons KS, Kohn N, et al. (2007) An evaluation of the vitamin D3 content in fish: Is the vitamin D content adequate to satisfy the dietary requirement for vitamin D? J Steroid Biochem Mol Biol 103: 642-644.
8. Alasalvar C, Taylor KDA, Zubcov E, Shahidi F, Alexis M (2002) Differentiation of cultured and wild sea bass (Dicentrarchus labrax): total lipid content, fatty acid and trace mineral composition. Food Chem 79: 145-150.

9. Carvalho ML, Santiago S, Nunes ML (2005) Assessment of the essential element and heavy metal content of edible fish muscle. Anal Bioanal Chem 382: 426-432.

10. Innis SM (2007) Dietary (n-3) fatty acids and brain development. J Nutr 137: 855-859.

11. Patrick RP, Ames BN (2015) Vitamin D and the omega-3 fatty acids control serotonin synthesis and action, part 2: relevance for ADHD, bipolar disorder, schizophrenia, and impulsive behavior. FASEB J 29: 2207-2222.

12. Kristensen SD, Iversen AM, Schmidt EB (2001) n-3 polyunsaturated fatty acids and coronary thrombosis. Lipids 36: 79-82.

13. Mori TA, Beilin LJ, Burke V, Morris J, Ritchie J (1997) Interactions between dietary fat, fish, and fish oils and their effects on platelet function in men at risk of cardiovascular disease. Arterioscler Thromb Vasc Biol 17: 279-286.

14. Nasopoulou C, Smith T, Detopoulou M, Tsikrika C, Papaharisis L, et al. (2014) Structural elucidation of olive pomace fed sea bass (Dicentrarchus labrax) polar lipids with cardioprotective activities. Food Chem 145: 1097 1105.

15. Nasopoulou C, Karantonis HC, Perrea DN, Theocharis SE, lliopoulos DG, et al. (2010) In vivo anti-atherogenic properties of cultured gilthead sea bream (Sparus aurata) polar lipid extracts in hypercholesterolaemic rabbits. Food Chem 120: 831-836.

16. Nasopoulou C, Tsoupras AB, Karantonis HC, Demopoulos CA, Zabetakis I (2011) Fish polar lipids retard atherosclerosis in rabbits by down-regulating PAF biosynthesis and up-regulating PAF catabolism. Lipids Health Dis 10: 213.

17. Kim SK, Mendis E (2006) Bioactive compounds from marine processing byproducts - A review. Food Res Int 39: 383-393.

18. Usydus Z, Szlinder-Richert J (2012) Functional Properties of Fish and Fish Products: A Review. Int J Food Properties 15: 823-846.

19. Papes F, Surpili MJ, Langone F, Trigo JR, Arruda P (2001) The essential amino acid lysine acts as precursor of glutamate in the mammalian central nervous system. FEBS Lett 488: 34-38.

20. Civitelli R, Villareal DT, Agnusdei D, Nardi P, Avioli LV, et al. (1992) Dietary L-lysine and calcium metabolism in humans. Nutrition 8: 400-405.

21. Schmid A, Walther B (2013) Natural vitamin D content in animal products. Adv Nutr 4: 453-462.

22. Rayman MP (2000) The importance of selenium to human health. Lancet 356: 233-241.

23. Sila A, Bougatef A (2016) Antioxidant peptides from marine by-products: Isolation, identification and application in food systems. A review. Journal of Functional Foods 21: 10-26. 